
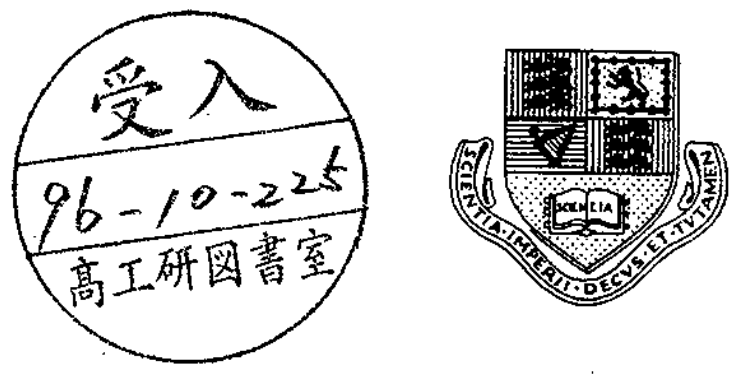

\title{
IMPERIAL COLLEGE OF SCIENCE, TECHNOLOGY AND MEDICINE
}

\author{
High Energy Physics Group
}

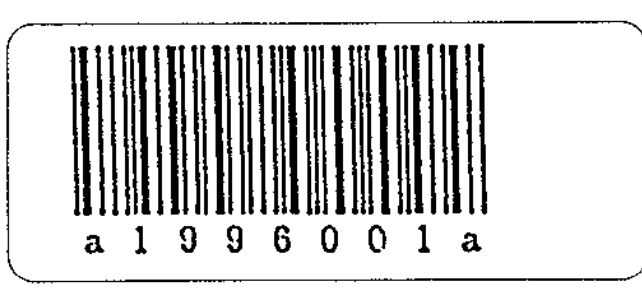

\section{Cherenkov-light detectors for LHC-B: an application for Hybrid Photodetectors}

\author{
D Websdale
}

On behalf of the LHC-B Collaboration

\begin{abstract}
The Ring Imaging Cherenkov detectors proposed for the CERN LHC-B experiment will need photon detectors with challenging specifications. Single photon sensitivity, millimetre precision and timing resolution adequate to resolve the LHC bunch-crossing period of $25 \mathrm{~ns}$ are required. The Hybrid Photodetector offers a promising solution.
\end{abstract}

Paper presented at "New Developments in Photodetection", Beaune, France, 1996. 


\title{
Cherenkov-light detectors for LHC-B: an application for Hybrid Photodetectors *
}

\author{
David Websdale, Blackett Laboratory, Imperial College, London, UK. For the LHC-B Collaboration
}

The Ring Imaging CHerenkov detectors proposed for the CERN LHC-B experiment will need photon detectors with challenging specifications. Single photon sensitivity, millimetre precision and timing resolution adequate to resolve the LHC bunch-crossing period of $25 \mathrm{~ns}$ are required. The Hybrid Photodetector offers a promising solution.

\section{INTRODUCTION}

The LHC-B experiment [1] has been proposed for a study of matter-antimatter asymmetry at the CERN LHC. Precision measurements of CPviolation using several decay modes of B-hadrons will require particle identification of the decay products over a wide momentum range. This will be achieved using Ring Imaging CHerenkov (RICH) detectors [2].

In this paper, we describe the main features of the proposed RICH detectors and present the results from a simulation of their performance. The operating conditions in the LHC-B experiment impose challenging requirements. Hybrid Photon Detectors (HPD's) are proposed as the favoured candidate device to detect the Cherenkov photons. Finally, we outline the $R \& D$ programme, now underway, to demonstrate experimentally the feasibility of our proposed detectors.

\section{LHC-B RICH: Concept}

Charged particles traversing a transparent material, of refractive index $n$, emit Cherenkov radiation into a cone of half-angle, $\theta_{c}=\cos ^{-1}(1 / \beta n)$ where $\beta c$ is the particle's velocity. When the particle's momentum is also known its mass (and hence the particle identity) can be determined.

To achieve $\pi / \mathrm{K}$ identification over the momentum range $1-150 \mathrm{GeV} / c$ three radiators will be needed. Their characteristics are summarised in Table 1. In the proposed LHC-B detector [1] the $\mathrm{CF}_{4}$ gas radiator is contained in a vessel located downstream of the spectrometer magnet where it is used to identify high momentum particles. The aerogel and $\mathrm{C}_{4} \mathrm{~F}_{10}$ gas are contained in the same vessel, upstream of the magnet and straddling the beam pipe as shown in Fig. 1.

\footnotetext{
-Paper presented at "New Developments in Photodetection", Beaune. France, 1996.
}

\begin{tabular}{|l||c|c|c|}
\hline Radiator & Aerogel & $\mathrm{C}_{4} \mathrm{~F}_{10}$ & $\mathrm{CF}_{4}$ \\
\hline \hline Refr. Index: $n$ & 1.03 & 1.0014 & 1.0005 \\
\hline Max. Angle: $\theta_{c}$ & 0.240 & 0.053 & 0.030 \\
\hline$p_{\text {threshold }}(\mathrm{GeV} / c): \pi$ & 0.6 & 2.7 & 4.6 \\
$p_{\text {threshold }}(\mathrm{GeV} / c): \mathrm{K}$ & 2.0 & 9.4 & 16.3 \\
\hline
\end{tabular}

Table 1

Characteristics of the radiators for LHC-B RICH detectors.

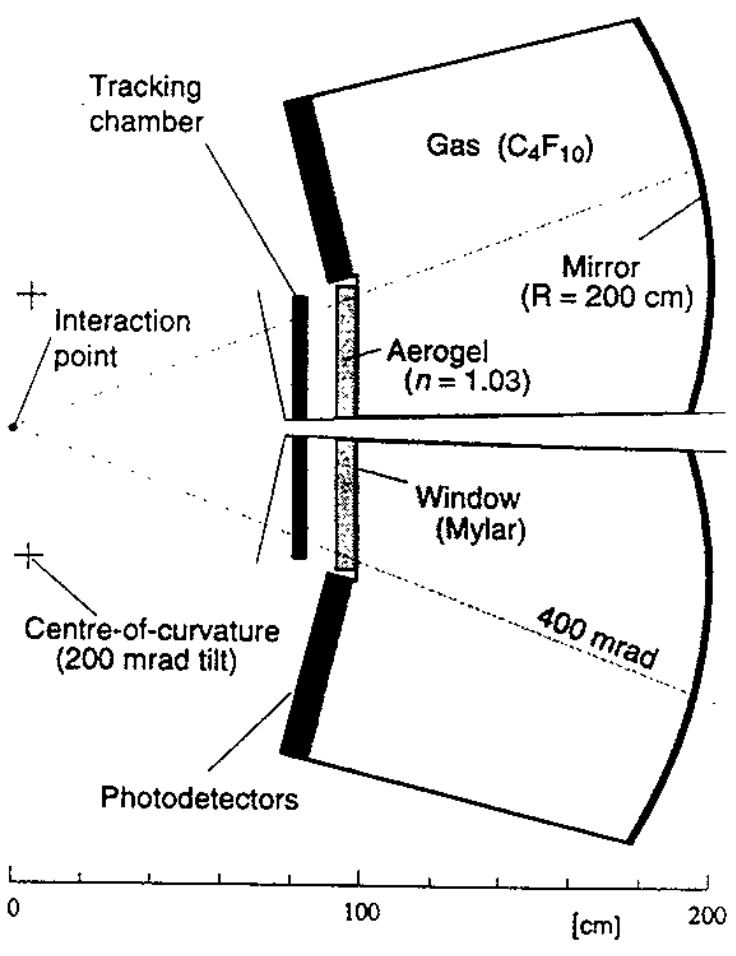

Figure 1. Layout of the combined Aerogel and gas $\mathrm{RICH}$ detector.

Particles entering this RICH detector vessel traverse $5 \mathrm{~cm}$ of aerogel and $100 \mathrm{~cm}$ of the $\mathrm{C}_{4} \mathrm{~F}_{10}$ radiator. Cherenkov photons emitted from aerogel and gas radiators are focussed into ring images by the 


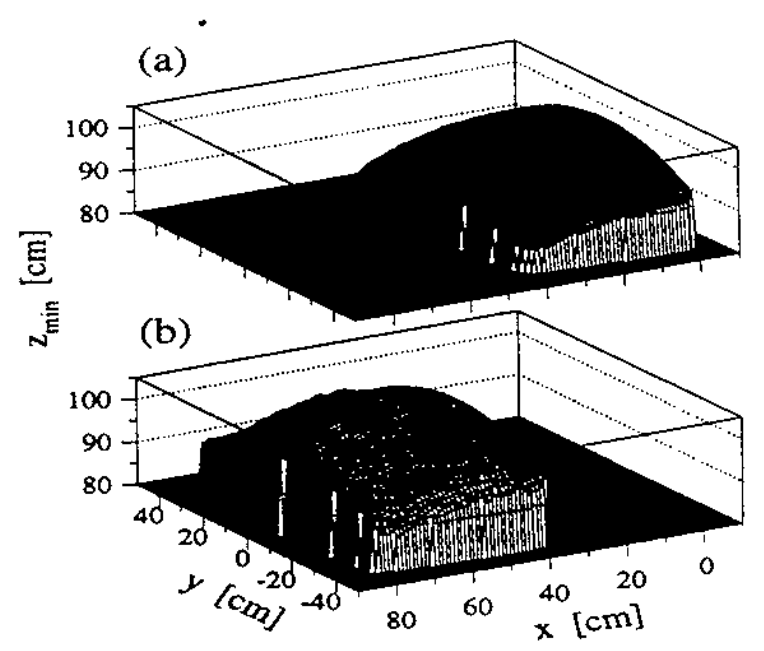

large spherical mirrors shown. The ring radii determine the corresponding Cherenkov emission angles, $\theta_{c}$. In the conventional RICH detector geometry [2], the centre of mirror curvature is located at the interaction point. This would require the photon detectors within the LHC-B spectrometer acceptance, resulting in an unacceptable quantity of material in the path of the particles. By dividing and tilting the spherical mirror as shown in Fig. 1, the detectors can be positioned at each side, beyond the spectrometer acceptance $( \pm 400 \mathrm{mrad}$ with respect to the $\mathrm{LHC}$ beam).

\section{RICH performance studies}

The performance of the combined aerogel-gas RICH detector has been evaluated using a Monte Carlo simulation. First, the precision in reconstruction of the Cherenkov emission angle was estimated. This determines the $\pi / K$ separation performance. Secondly, the problem of pattern recognition was studied. Cherenkov radiation is a weak source and the numbers of detected photons associated with each ring are limited. Finding the Cherenkov rings among the many imaged at the detector surface by the high multiplicity of charged particles produced in a typical LHC interaction is not a trivial task.

\subsection{Precision in reconstruction of $\theta_{c}$}

Four components contribute to the spatial precision of the Cherenkov photons at the ring image.

\section{Geometry of the optical system}

The standard geometry of a RICH detector, with the particle trajectory along the mirror axis, results in a spherical focal surface. Tilting the mirror produces an irregular focal surface (see Fig. 2) whose aberrations can be partially corrected by optimizing the location of the detector plane to fit this focal surface.

\section{Uncertainty of photon emission point}

Charged particles traverse $100 \mathrm{~cm}$ of the gas radiator. The emission point of the Cherenkov photon cannot be reconstructed from the impact point of the photon at the detector. Taking the central point on the track as emission point results in an r.m.s error, $\sigma_{\theta}=0.6 \mathrm{mrad}$, in the reconstructed Cherenkov angle.

\section{Chromatic dispersion in $\mathrm{C}_{4} \mathrm{~F}_{10}$}

Cherenkov photons are emitted uniformly across the energy spectrum and it is desirable to detect as many of these photons as possible. A photon detector with a bialkali photocathode on a quartz window has quantum efficiency $\sim 20 \%$ for $2<E_{\gamma}<5.5 \mathrm{eV}$. However, over this range the refractive index of the

Figure 2. Effect of tilting the mirror. (a) Spherical focal surface with coaxial mirror, (b) displacement and aberration resulting from tilted mirror.

$\mathrm{C}_{4} \mathrm{~F}_{10}$ radiator varies from $1.00135-1.00145$. If the photon energy is not measured (as is the case here) this chromatic aberration results in an uncertainty in the reconstructed Cherenkov emission angle $\theta_{c}$. such that the r.m.s precison, $\sigma_{\theta}=0.6 \mathrm{mrad}$.

\section{Photon detector spatial resolution}

It is undesirable if the error due to the pixel size of the photon detector exceeds the geometric and chromatic errors, which contribute to the precision in $\theta_{c}$. With a pixel dimension of $2 \times 2 \mathrm{~mm}^{2}$ the overall spatial resolution function resulting from all contributions is approximately Gaussian, with $\sigma_{\theta}=$ $1.0 \mathrm{mrad}$.

\subsection{Photo-electron yield}

The number of photo-electrons detected from a charged particle traversing $L \mathrm{~cm}$ of radiator is

$$
N_{\mathrm{pe}}=370 \varepsilon L \int \sin ^{2} \theta_{\mathrm{c}} Q(E) d E,
$$

where $\varepsilon$ is a geometrical factor which accounts for incomplete photon detector coverage. If the quantum efficiency integral is calculated for a bialkali photocathode on a quartz window then $N_{\text {pe }}=60$ is estimated for the $100 \mathrm{~cm} \mathrm{C}_{4} \mathrm{~F}_{10}$ radiator and the intrinsic precision on the Cherenkov angle for the track is $\sigma_{\theta} / \sqrt{N_{\text {pe }}} \sim 0.12 \mathrm{mrad}$.

\subsection{Silica Aerogel radiator}

The use of silica aerogel in threshold Cherentov detectors is an established technique. Ring imaging is new. Images have been observed by photographing the superposed rings from many tracks [3]. First results using electronic detectors have been 
obtained ${ }^{2}$ using a $10 \mathrm{GeV} \pi$ beam at CERN with a $3 \mathrm{~cm}$ thick aerogel tile ${ }^{3}$. Fig. 3 displays the re-

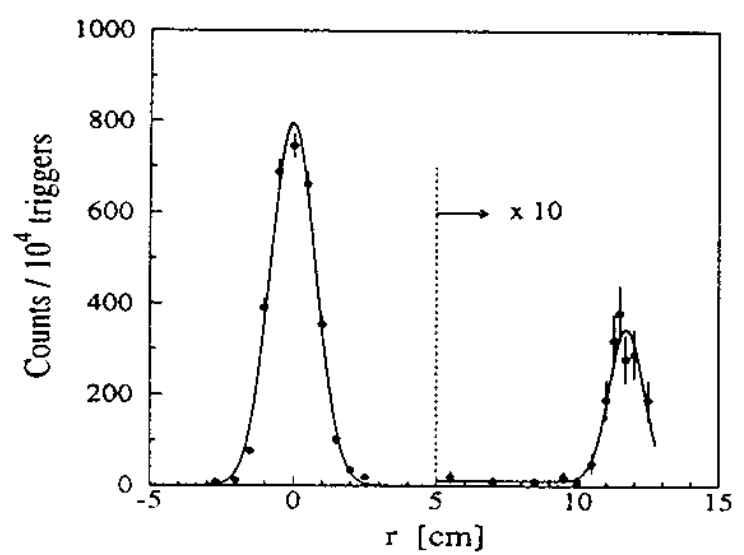

Figure 3. Scan across diameter of concentric air and aerogel rings. Note the very low background due to scattered photons in the gap between rings.

sult of a scan, using a one inch diameter PM tube, across the diameter of the expected ring. The peak on the left corresponds to the (unresolved) ring of Cherenkov radiation from air. The right-hand peak is the aerogel ring, whose radius is compatible with its measured refractive index, $n=1.03$. The $3 \mathrm{~cm}$ radiator yields, on average, 14 detected photoelectrons. The tests are still in progress and final results will be reported elsewhere [4].

\subsubsection{Silica Aerogel in LHC-B RICH}

Rayleigh scattering in the aerogel results in a loss of photons from the ring image and a uniformly scattered background. The effect increases as $E_{\gamma}^{4}$ so it will be necessary to absorb the UV photons $\left(E_{\gamma}>3.5 \mathrm{eV}\right)$. Using a filter at the downstream end of a $5 \mathrm{~cm}$ aerogel tile we estimate $N_{\text {pe }} \sim 15$ detected photo-electrons on the ring (and 5 scattered elsewhere). The corresponding contributions to the $\theta_{c}$ resolution are listed in Table 2 . The chromatic error is the main contribution and the overall resolution function for one track has an r.m.s $\sigma_{\theta} \sim 0.4 \mathrm{mrad}$.

\subsection{Particle identification performance}

The ability to distinguish $\pi$ 's from K's of a given momentum $p$, depends on a comparison of the measured Cherenkov angle $\theta_{c}$ with that expected for a $\pi$ and for a $\mathrm{K}$ traversing the radiator. The curves on Fig. 4 display (for each of three radiators) the $\pi / \mathrm{K}$ separation, measured in units of $\sigma_{\theta}$, as a function of

\footnotetext{
${ }^{2}$ In collaboration with INFN-Bari, Lecce, Milano and Roma.
} ${ }^{3}$ Supplied by the KEK laboratory, Japan.

\begin{tabular}{|l||c|c|}
\hline Radiator & $\mathrm{C}_{4} \mathrm{~F}_{10}$ & Aerogel \\
\hline \hline Emission point error & 0.6 & 0.3 \\
\hline Chromatic error & 0.6 & 1.2 \\
\hline Pixel error & 0.6 & 0.6 \\
\hline Total error / p.e. & 1.0 & 1.4 \\
\hline Total error /track & 0.12 & 0.4 \\
\hline
\end{tabular}

\section{Table 2}

Contributions to r.m.s precision (mrad) in reconstruction of the Cherenkov emission angle $\theta_{c}$.

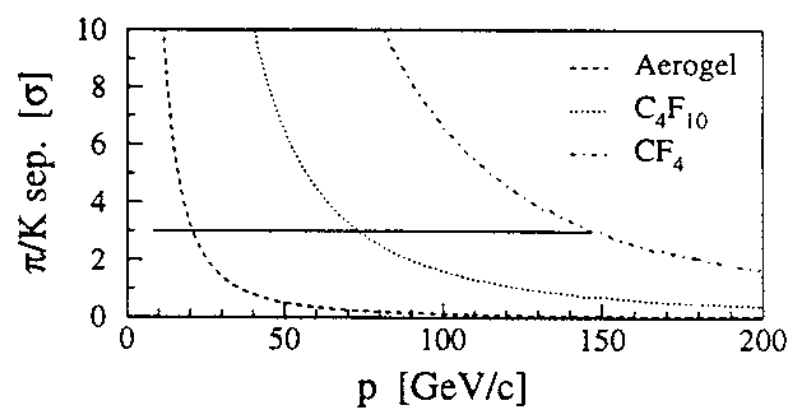

Figure 4. Upper momentum limit for $\pi / K$ separation for the three radiators.

particle momentum. The horizontal line indicates $\pi / \mathrm{K}$ separation with $3 \sigma$ significance and hence an upper limit to the momentum at which $\pi$ and $\mathrm{K}$ particles can confidently be distinguished.

This measurement is possible only if the ring images are correctly identified. In practice an LHC interaction results in many $(\sim 50)$ particles traversing the RICH detectors. A typical simulated event is shown in Fig. 5 . It contains 46 tracks, 25 of which exceed the $\mathrm{C}_{4} \mathrm{~F}_{10}$ threshold (and hence produce both gas and aerogel rings). A total of more than 1000 photo-electron "hits" have to be assigned. In spite of this daunting proposition, the preliminary results from the pattern recognition algorithms are encouraging, e.g. the gas rings from 23 tracks (out of the 25 in Fig. 5) were correctly reconstructed.

\section{Requirements for photon detectors}

Based on the results of these simulations we can list the requirements of the LHC-B RICH photon detectors:

- Single photon sensitivity is essential.

- High quantum efficiency in visible/UV range $(220-600 \mathrm{~nm})$. 


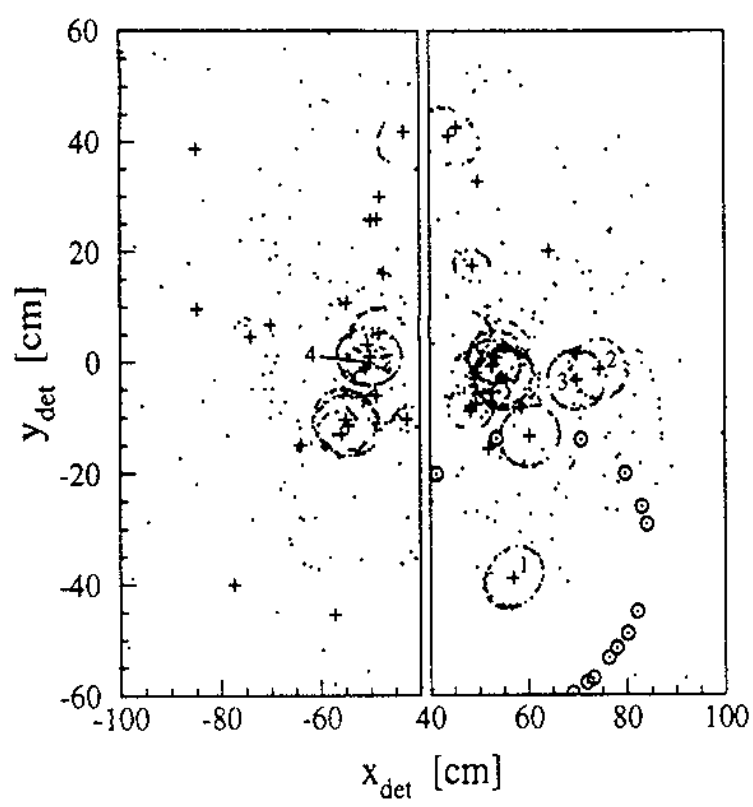

Figure 5. Photon "hits" in the upstream RICH, for a typical $\mathrm{LHC}-\mathrm{B}$ event. For illustration, the passage of each track is indicated by a cross and the hits from the aerogel ring for track 1 are circled.

- Low noise count.

- Fast: the LHC bunch crossing period is $25 \mathrm{~ns}$.

- Pixel size: $2 \times 2 \mathrm{~mm}^{2}$ for the Aerogel $/ \mathrm{C}_{4} \mathrm{~F}_{10}$ detector, $4 \times 4 \mathrm{~mm}^{2}$ for the $\mathrm{CF}_{4}$ detector.

- Cover large area: $\sim 4 \mathrm{~m}^{2}$ with high fractional coverage.

An HPD device, in which the photo-electrons from a bialkali-type photocathode are accelerated to bombard a segmented silicon detector, appears as the most promising candidate. If we assume that HPD's with $100 \mathrm{~mm}$ photocathode diameter could be packed so as to cover a fraction $80 \%$ of the focal surface, then we would require approximately 400 HPD tubes, with a total of $4 \times 10^{5}$ pixels. Such devices are not available today, and will require a vigorous development programme.

\section{R\&D in progress for LHC-B RICH}

Before the LHC-B RICH detectors can be constructed several of the ideas need to be proven and prototyped. We summarize below a four-pronged $R \& D$ programme, which is already underway.

\section{ISPA tube}

The Imaging Silicon Pixel Array is an HPD with $10^{3}$ pixels and read-out chip enclosed within the vacuum tube. While not optimized for the LHCB RICH application it permits testing many of the required features. Results are reported at this conference by T.Gys [5].

\section{"In-House" HPD development}

In another presentation at this conference P.Martinengo [6] reports the first results from tests on an HPD, built at CERN, using a CsI photocathode and a silicon pixel detector. The single photon sensitivity is well demonstrated. This programme aims to demonstrate the elements which will permit the development of an HPD which satisfies the LHC-B requirements.

\section{Simulations}

The Monte Carlo simulations of the LHC-B RICH detector and the pattern recognition algorithms, as described in section 3 , will be pursued and improved. These will include simulations of the prototype HPD detectors to compare with experimental measurements.

\section{Prototype LHC-B RICH}

A prototype RICH detector will be tested in charged particle beams during 1997. The detector will be equipped with commercial HPD's ${ }^{4}$ and aims to provide a "system" test and demonstration of the following features:

- Detection of Cherenkov rings from gas and aerogel radiators using HPD's (and other photon detectors).

- Measurement of detected photon yield, single photon sensitivity, spatial precision and noise.

- Measurememts of optical properties of mirrors, UV filters, Rayleigh scattering, etc.

- Test read-out of HPD's at LHC speed $(<$ $25 \mathrm{~ns})$.

- Measure the effects due to particles traversing the HPD's.

\section{Conclusions}

Particle identification is a crucial feature of the LHC-B experiment. Our studies show that the requirements can be met using RICH detectors with aerogel and fluorocarbon gas radiators. The photon detectors require $\mathrm{mm}$ spatial precision, timing resolution better than $25 \mathrm{~ns}$, single photon sensitivity and good quantum efficiency in the visible and UV spectrum. HPD's are seen as the most promising candidate photon detectors and a comprehensive 
$R \& D$ programme is underway to demonstrate the feasibility of a RICH detector with an image area covering $4 \mathrm{~m}^{2}$ equipped with HPD's.

\section{Acknowledgements}

The work reported here has been selected from studies and tests undertaken by members of the LHC-B RICH team. The Aerogel tests were performed in collaboration with groups from the universities of Bari, Lecce, Milano and Roma. We gratefully acknowledge the KEK laboratory, Japan, for supply of the aerogel samples tested in the beam. The author thanks the organising committee for planning an enjoyable and successful conference.

\section{REFERENCES}

1. Lol for LHC-B. CERN/LHCC 95-5 (1995)

D.Websdale. Nucl. Physics B (Proc.Suppl) 50 (1996) 333.

2. T.Ypsilantis and J.Seguinot, Nucl. Instr. and Meth. A343 (1994) 30.

3. D.Fields et al.. Nucl. Instr. and Meth. A349 (1994) 43 i.

4. V.Manzari et al. Submitted for publication in Nucl. Instr and Meth. A.

5. Position-sensitive single-photon detection with a hybrid Silacon Pixel Array tube.

T.Gys, at this conference.

6. A silicon pad detector for HPD's.

P.Martinengo. at this conference. 